\title{
Insuficiência venosa crônica e uso de meia elástica de compressão graduada: uma análise sobre a adesão ao tratamento em pacientes do SUS
}

\author{
Chronic venous insufficiency and graduated compression stockings: analysis of public \\ health system patients' adherence to treatment
}

Francisco Eduardo Coral'1,2 (D), Giovanna Golin Guarinello² (D), Alice Pavanatto Cavassola', Ana Luiza Moraes Rocha', Marina Mosele Guidi', Hudson Pires ${ }^{1}$

\begin{abstract}
Resumo
Contexto: A insuficiência venosa crônica é uma doença de alta prevalência mundial, podendo chegar a até $80 \%$ da população. Sua incidência aumenta com a idade e é mais frequente no sexo feminino. Das opções terapêuticas, destacase a terapia compressiva, sendo a principal o uso de meia elástica de compressão graduada, considerado o tratamento básico para a insuficiência venosa crônica independentemente da classificação clínica do paciente. Na prática clínica, o resultado da terapia é prejudicado pela não adesão ao uso da meia. Objetivos: Avaliar a taxa de adesão ao uso da meia elástica de compressão graduada, assim como compreender a problemática da não aderência ao tratamento. Métodos: Estudo observacional transversal, realizado entre junho de 2017 até janeiro de 2019, mediante aplicação de questionário aos pacientes em ambulatório de cirurgia vascular do Sistema Único de Saúde (SUS), em um hospitalescola, em Curitiba, no estado do Paraná (PR). Os dados foram analisados com o programa computacional IBM SPSS Statistics v.20.0. Resultados: Foram analisados 240 pacientes. A média de idade foi de 57,5 $\pm 12,9$ (22-86); 84,2\% eram do sexo feminino. Do total de pacientes analisados, 106 (44,2\%) não aderiram ao uso das meias. As justificativas para o não uso foram: questão financeira, dor, desconhecimento da necessidade, calor e outras. Conclusões: A taxa de adesão encontrada no presente estudo foi de $55,8 \%$, e o principal motivo para o não uso foi a questão financeira.
\end{abstract}

Palavras-chave: meias de compressão; insuficiência venosa; cooperação e adesão ao tratamento.

\begin{abstract}
Background: Chronic venous insufficiency $(\mathrm{CVI})$ is a pathology of great importance due to its high worldwide prevalence, affecting up to $80 \%$ of the population. Its incidence increases with age and is more frequent in females. One of the most important treatment options is compression therapy and the main method employed is wearing graduated compression stockings, which is considered the basic treatment for CVI regardless of the patient's clinical classification. In clinical practice, treatment outcomes are impaired by patients not wearing the stockings properly. Objectives: To analyze the rate of adherence to wearing graduated compression stockings and to understand the problem of treatment non-adherence. Methods: Cross-sectional observational study conducted from June 2017 to January 2019, based on administration of questionnaires to patients at a SUS vascular surgery clinic at a teaching hospital, in Curitiba, PR, Brazil. Data were analyzed using the IBM SPSS Statistics v.20.0 computer program. Results: 240 patients were analyzed. Mean age was $57.5 \pm 12.9(22-86)$ and $84.2 \%$ of the sample were female. 106 of the 240 patients analyzed (44.2\%) were non-adherent with wearing compression stockings. Reasons for not wearing the stockings were: financial; pain; ignorance of the need to wear them; heat; and others. Conclusions: The adherence rate observed in the present study was $55.8 \%$ and the most prevalent reason for not wearing stockings was financial.
\end{abstract}

Keywords: compression stockings; venous insufficiency; treatment adherence and compliance.

Como citar: Coral FE, Guarinello GG, Cavassola AP, Rocha ALM, Guidi MM, Pires H. Insuficiência venosa crônica e uso de meia elástica de compressão graduada: uma análise sobre a adesão ao tratamento em pacientes do SUS. J Vasc Bras. 2021;20:e20200034. https://doi.org/10.1590/1677-5449.200034

\footnotetext{
${ }^{1}$ Pontifícia Universidade Católica do Paraná - PUCPR, Curitiba, PR, Brasil.

${ }^{2}$ Hospital Santa Casa de Misericórdia, Curitiba, PR, Brasil.

Fonte de financiamento: Nenhuma.

Conflito de interesse: Os autores declararam não haver conflitos de interesse que precisam ser informados.

Submetido em: Junho 16, 2020. Aceito em: Fevereiro 01, 2021
}

O estudo foi realizado no Hospital da Irmandade da Santa Casa de Misericórdia de Curitiba, Curitiba, PR, Brasil. 


\section{INTRODUÇÃO}

A insuficiência venosa crônica (IVC) dos membros inferiores é uma afecção bastante comum ${ }^{1}$, que tem sua prevalência aumentada com a idade e apresenta uma incidência maior no sexo feminino ${ }^{2,3}$. A sua fisiopatologia envolve a presença de hipertensão intravenosa de longa duração $0^{4,5}$ resultante de mau funcionamento do sistema venoso devido a uma incompetência valvular, com ou sem obstrução do fluxo venoso ${ }^{2,5,6}$. Os resultados finais são o refluxo sanguíneo e a estase venosa ${ }^{2}$.

O quadro clínico é variável ${ }^{2}$, desde alterações estéticas até a presença de sintomas e sinais que provocam morbidade significativa e perda de dias de trabalho, como a presença de úlceras venosas ${ }^{1,7}$. O diagnóstico da IVC é clínico, baseado nos dados da anamnese e do exame físico ${ }^{2,8}$.

A classificação clínica da IVC é composta por seis classes. Assim, 0 representa casos em que não há sinais visíveis ou palpáveis de doença venosa; 1 e 2, doença inicial; e os demais, 3, 4 e 5, característicos de doença mais avançada com edema (3), alterações de pele (4) e úlceras venosas cicatrizadas (5) ou ativas $(6)^{2,5,6,9}$.

Das opções terapêuticas, destaca-se a terapia compressiva, considerada o tratamento básico para a IVC independentemente da classificação clínica do paciente ${ }^{4}$. Entre as terapias compressivas, está a meia elástica de compressão graduada (MECG), um método simples e de alta eficácia9. Ela atua mecanicamente provocando a diminuição do diâmetro das veias com redução do refluxo e da pressão venosa e o aumento da velocidade e do retorno venoso ${ }^{3}$. Assim, ela melhora a função da bomba muscular e reduz a estase sanguínea ${ }^{1-3}$. Os benefícios são limitados ao período do uso, uma vez que, após a sua retirada, o efeito hemodinâmico provocado sobre o membro inferior cessa em aproximadamente 1 hora $^{10}$.

Na prática clínica, o resultado da terapia é prejudicado pela baixa adesão ao tratamento, prejudicando os resultados $^{1,9}$. O objetivo deste trabalho foi avaliar a taxa de adesão ao uso da meia elástica de compressão graduada, assim como compreender a problemática da não aderência ao tratamento.

\section{MÉTODO}

Estudo aprovado pelo Comitê de Ética em Pesquisa (CEP) da Pontifícia Universidade Católica do Paraná (PUCPR) - CAAE: 56980816.8.0000.0020. Parecer consubstanciado número: 2.955 .862 . Trata-se de estudo observacional transversal realizado entre junho de 2017 e janeiro de 2019, composto por 240 pacientes portadores de IVC com mais de 18 anos de idade, de ambos os sexos, atendidos no ambulatório de Cirurgia Vascular do Sistema Único de Saúde (SUS) de um hospital-escola no município de Curitiba, Paraná.
Os critérios de exclusão foram presença de doença arterial obstrutiva crônica de membros inferiores, insuficiência cardíaca descompensada, presença de processo inflamatório ou infecioso agudo nos membros inferiores e perda de seguimento. Todos os participantes do estudo estavam cientes da pesquisa e assinaram o termo de consentimento livre e esclarecido.

A coleta de dados ocorreu durante as consultas de retorno dos pacientes em acompanhamento por IVC no ambulatório de Cirurgia Vascular. As informações foram coletadas por meio de um questionário (Figura 1), sendo que o único campo preenchido pelo médico foi a classificação clínica, os demais tendo sido respondidos pelo paciente.

A adesão foi definida como o uso da meia por pelo menos 7 horas diárias em 5 dias na semana, e o paciente foi classificado de acordo com a classe clínica mais avançada. Os dados foram armazenados em planilha do Microsoft Excel ${ }^{\mathbb{B}}$ (Microsoft Corporation, Albuquerque, Novo México, EUA). Os resultados obtidos no estudo foram descritos por médias, desvios padrões e valores mínimos e máximos (variáveis quantitativas) e por frequências e percentuais (variáveis categóricas). A associação entre duas variáveis categóricas foi avaliada considerando-se o teste de qui-quadrado. Valores de $\mathrm{p}<0,05$ indicaram significância estatística. Os dados foram analisados com o programa computacional IBM SPSS Statistics v.20.0 (IBM Corp, Armonk, Nova Iorque, EUA).

\section{RESULTADOS}

Foram avaliados um total de 240 pacientes, a média de idade foi de 57,5 $\pm 12,9$ (22-86). Do total, $202(84,2 \%)$ eram do sexo feminino, e $38(15,8 \%)$, do sexo masculino. Dos pacientes analisados, $177(73,8 \%)$ relataram fazer o uso da MECG, e $63(26,2 \%)$ não faziam. As justificativas dos pacientes para o não uso da MECG foram questão financeira (33,3\%), dor $(28,6 \%)$, desconhecimento da necessidade (19\%), calor (6,35\%), não conseguir colocar com ou sem ajuda $(6,34 \%)$ e outras $(6,35 \%)$ (edema, estética, não querer usar e sensação de piora dos sintomas).

Nos pacientes que utilizavam, o tempo médio de uso diário em horas foi de $11,1 \pm 2,8$ (2-18), e a média de dias na semana foi de $6,0 \pm 1,0$ (1-7). A distribuição em idade, sexo, renda mensal, classificação clínica, uso da MECG e adesão é mostrada na Tabela 1.

Como já citado, o paciente aderente deveria utilizar a MECG por um período contínuo de no mínimo 7 horas diárias por 5 dias na semana, considerando um período padrão de horário de trabalho. Dessa forma, apesar de relatarem fazer o uso da MECG, $43(17,9 \%)$ pacientes também foram considerados como não aderentes ao tratamento. Sendo assim, do total de 240 pacientes, 134 foram considerados como aderentes ao uso de MECG, correspondendo 


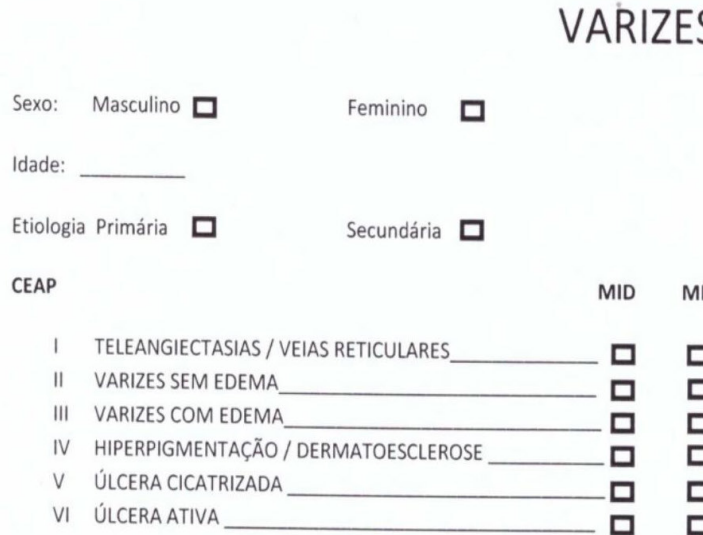

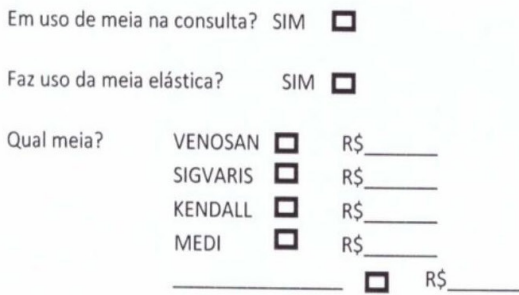
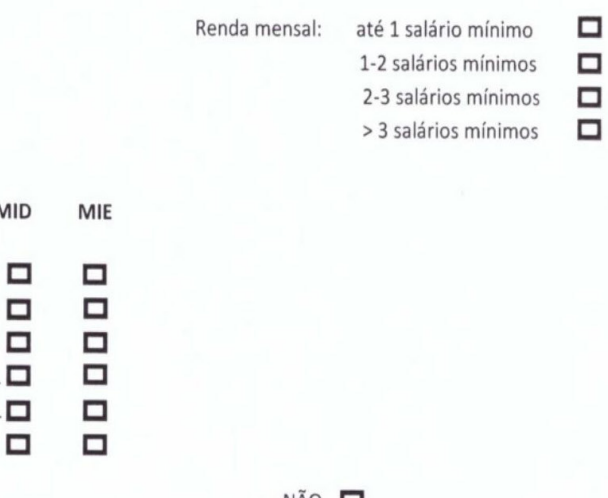

Motivo de ter comprado a marca:

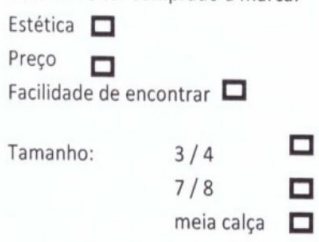

Quando coloca a meia:
Manhã $\square$
Tarde $\square$
Noite $\square$
Quando dói $\square$

Quanto tempo, por dia, faz uso da meia: (horas)

Quantos dias na semana faz uso da meia:

$\begin{array}{ll}1 \text { 口 } & 5 \text { 口 } \\ 2 \text { 口 } & 6 \text { 口 } \\ 3 & 7\end{array}$

Tempo que demorou para iniciar uso da meia:

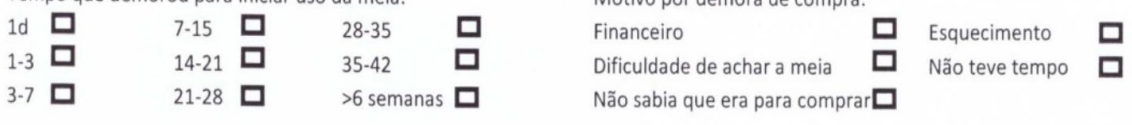

Compressão:

$15-20 \mathrm{mmHg}$

20-30 mmHg

$30-40 \mathrm{mmHg}$

Figura 1. Questionário aplicado nos pacientes do estudo.

a $55,8 \%$, com intervalo de confiança de $95 \%$ dado por $49,6 \%$ a $62,1 \%$.

Para a análise de cada variável, foram considerados os dados válidos (não perdidos), pois quatro pacientes não informaram a renda mensal e um paciente não foi classificado clinicamente. Em relação aos fatores associados à adesão, não houve diferença estatisticamente significante entre idade, sexo e classificação clínica $(p>0,05)$, conforme demonstrado na Tabela 2. A avaliação da associação entre a renda 
Tabela 1. Análise descritiva geral.

\begin{tabular}{lccc}
\hline \multicolumn{1}{c}{ Variável } & n válido & Classificação & Resultado \\
\hline Idade (anos) & 240 & & $57,5 \pm 12,9(22-86)$ \\
& & 20 a 40 & $31(12,9)$ \\
& & 40,1 a 65 & $130(54,2)$ \\
Sexo & & $>65$ & $79(32,9)$ \\
Renda mensal & 240 & Feminino & $202(84,2)$ \\
& & Masculino & $38(15,8)$ \\
& 236 & Até 1 SM & $99(41,9)$ \\
Classificação clínica & & $1-2$ SM & $97(41,1)$ \\
& & $2-3$ SM & $29(12,3)$ \\
& & $>3$ SM & $11(4,7)$ \\
& & I & $15(6,3)$ \\
& & II & $57(23,8)$ \\
Faz uso da meia & & III & $98(41)$ \\
& & IV & $35(14,6)$ \\
Adesão & & V & $20(8,4)$ \\
& & VI & $14(5,9)$ \\
& & Não & $63(26,2)$ \\
& & Sim & $177(73,8)$ \\
& & Não & $106(44,2 \%)$ \\
& & Sim & $134(55,8 \%)$ \\
\hline
\end{tabular}

Descrito por média \pm desvio padrão (mínimo-máximo) ou por frequência (percentual). SM = salário mínimo.

Tabela 2. Análise de variáveis $\times$ adesão.

\begin{tabular}{|c|c|c|c|}
\hline \multirow{2}{*}{ Variável } & \multicolumn{2}{|c|}{ Adesão } & \multirow{2}{*}{ Total } \\
\hline & Sim & Não & \\
\hline \multicolumn{4}{|l|}{ Sexo } \\
\hline \multirow[t]{2}{*}{ Feminino } & 115 & 87 & 202 \\
\hline & $56,90 \%$ & $43,10 \%$ & \\
\hline \multirow[t]{2}{*}{ Masculino } & 19 & 19 & 38 \\
\hline & $50 \%$ & $50 \%$ & \\
\hline \multicolumn{4}{|c|}{ Valor de p: 0,478 (teste exato de Fisher, $\mathrm{p}<0,05$ ) } \\
\hline \multicolumn{4}{|c|}{ Idade } \\
\hline \multirow[t]{2}{*}{$20-40$} & 19 & 12 & 31 \\
\hline & $61,30 \%$ & $38,70 \%$ & \\
\hline \multirow[t]{2}{*}{$41-65$} & 71 & 59 & 130 \\
\hline & $54,60 \%$ & $45,40 \%$ & \\
\hline \multirow[t]{2}{*}{$>65$} & 44 & 35 & 79 \\
\hline & $55,70 \%$ & $44,30 \%$ & \\
\hline \multicolumn{4}{|c|}{ Valor de p: 0,797 (teste de qui-quadrado, $p<0,05$ ) } \\
\hline \multicolumn{4}{|c|}{ Classificação clínica } \\
\hline \multirow[t]{2}{*}{ I } & 7 & 8 & 15 \\
\hline & $46,60 \%$ & $53,40 \%$ & \\
\hline \multirow[t]{2}{*}{ II a III } & 85 & 70 & 155 \\
\hline & $54,80 \%$ & $45,20 \%$ & \\
\hline \multirow[t]{2}{*}{ IV a VI } & 42 & 27 & 69 \\
\hline & $60,90 \%$ & $39,10 \%$ & \\
\hline
\end{tabular}

Valor de p: 0,528 (teste de qui-quadrado, $\mathrm{p}<0,05$ ).
Tabela 3. Renda mensal $\times$ adesão.

\begin{tabular}{|c|c|c|c|}
\hline \multirow{2}{*}{ Renda } & \multicolumn{2}{|c|}{ Adesão } & \multirow{2}{*}{ Total } \\
\hline & Sim & Não & \\
\hline \multirow[t]{2}{*}{1 e $2 S M$} & 106 & 90 & 196 \\
\hline & $54 \%$ & $46 \%$ & \\
\hline \multirow[t]{2}{*}{$3 S M$} & 20 & 9 & 29 \\
\hline & $68,90 \%$ & $31,10 \%$ & \\
\hline \multirow[t]{2}{*}{$>3 \mathrm{SM}$} & 7 & 4 & 11 \\
\hline & $63,60 \%$ & $36,40 \%$ & \\
\hline
\end{tabular}

Valor de p: 0,162 (teste exato de Fischer, $\mathrm{p}<0,05$ ). SM = salário mínimo.

mensal e a adesão ao uso da MECG está demonstrada na Tabela 3, porém também não mostrou significância estatística $(\mathrm{p}>0,05)$.

\section{DISCUSSÃO}

Diversas literaturas médicas asseguram a grande eficácia ao tratamento da IVC com o uso da MECG, como, por exemplo, o ensaio clínico prospectivo multicêntrico randomizado e duplo-cego, realizado na França por Benigni et al. ${ }^{4}$, com 125 pacientes do sexo feminino, o qual demonstrou melhora significativa na sintomatologia da IVC em estágios clínicos iniciais com o uso de meias de baixa compressão (pressão no tornozelo de 10 a $15 \mathrm{mmHg}$ ) em comparação com meias placebo (pressão no tornozelo de 3 a $6 \mathrm{mmHg}$ ). Vayssairat et al. ${ }^{11}$ conduziram estudo semelhante, também na França, abrangendo 341 pacientes, no qual o resultado foi equivalente, com alívio significativo dos sintomas com a utilização de meias compressivas de mesma graduação.

No entanto, apesar das evidências positivas em relação ao uso de meias elásticas para o tratamento da IVC, seu uso é escasso ou inadequado. Os valores encontrados para adesão neste estudo, de 55,8\%, foram superiores aos da literatura. No estudo de Raju et al. ${ }^{12}$, realizado nos Estados Unidos, 63\% dos pacientes aos quais foi prescrito o uso da meia elástica não faziam o uso; os $37 \%$ restantes foram classificados como aderentes, independente de o uso ser regular ou não ${ }^{12}$. Ao analisar a população do México, Ayala-García et al. ${ }^{13}$ observaram que apenas $35,1 \%$ utilizavam a terapia compressiva.

Outro estudo realizado na Polônia, por Ziaja et al. ${ }^{14}$, encontrou uma percentagem de uso de $25,6 \%$ dos pacientes, sendo maior em pacientes mais velhos e que apresentavam uma classificação clínica mais avançada. Esse resultado corrobra o visto neste estudo, em que pacientes maiores de 65 anos eram mais aderentes, apesar de não haver significância estatística. 
Outro ponto a ser levado em consideração é que a amostra do presente estudo foi composta por pacientes em acompanhamento no ambulatório de Cirurgia Vascular, sendo esse um fator que provavelmente contribui para a maior adesão. Ayala-García et al. ${ }^{13}$ analisaram 168 pacientes que apresentavam IVC, em um ambulatório não específico da Cirurgia Vascular. Do total, apenas $59(35,1 \%)$ pacientes eram aderentes ao tratamento ${ }^{13}$. Além disso, foi observada uma diferença estatística naqueles que já tinham sido tratados de alguma forma para IVC, sendo esses 3,3 vezes mais prováveis de usar a meia, em comparação àqueles que nunca tiveram atenção médica prévia para doença $\operatorname{vascular}^{13}$.

A análise da não adesão ao tratamento e classificação dos participantes em aderentes e não aderentes, contudo, foi prejudicada pelo fato de não existir uma padronização quanto ao número de dias e de tempo em horas de uso para considerar o paciente aderente. Em seu artigo, Raju et al. ${ }^{12}$ explicaram que o conceito de compliance, por eles utilizado, é muito amplo, abrangendo uso regular, uso na maioria dos dias e uso infrequente ${ }^{12}$.

Ao levarmos em consideração os motivos de não uso, há uma grande divergência ao comparar com as populações de outros países. Por exemplo, no estudo de Raju et al. ${ }^{12}$, o principal motivo de não uso foi a incapacidade de determinar um motivo específico (30\%), seguido de não recomendado pelo médico, sensação de inefetividade do tratamento, aperto, dificuldade de colocar e calor. No presente estudo, os motivos de não uso foram principalmente questão financeira $(33,34 \%)$, dor $(18 \%)$ e desconhecimento da necessidade (12\%).

Com relação ao valor das meias elásticas, no estudo polonês realizado por Ziaja et al. ${ }^{14}, 33 \%$ dos pacientes relataram que não fizeram o uso devido ao alto custo, valor que coincide com o encontrado neste trabalho (33,3\%), enquanto, no estudo americano realizado por Raju et al. ${ }^{12}$, apenas $2 \%$ relataram essa justificativa. Apesar de apresentar valores similares nos três países, o presente estudo foi realizado apenas com pacientes do SUS, o que pode justificar esse fator como o principal motivo de não uso.

O presente estudo tem como limitações o fato de avaliar apenas pacientes do SUS, e não a população de uma forma mais homogênea. Ressalta-se a importância dos dados encontrados como auxílio aos angiologistas e cirurgiões vasculares no momento da prescrição das MECG como uma forma de prever e auxiliar nas dificuldades da adesão ao uso delas.
Ademais, vale enfatizar que o reforço na explicação feita em consultas provavelmente aumentaria a adesão dos pacientes. Alguns especialistas em terapia compressiva apontam a pobre educação do paciente como a raiz do problema de não adesão, pois diversos pacientes não entendem o correto uso e os benefícios da terapia compressiva ${ }^{15,16}$. O envolvimento do médico na educação do paciente, ao explicar o modo de uso e como vestir a meia, tende a aumentar a adesão e, portanto, a melhorar os benefícios e os resultados com a terapia compressiva.

\section{CONCLUSÃO}

A taxa de adesão encontrada no presente estudo foi de $55,8 \%$ e superior às taxas encontradas na literatura. Assim, inferimos e relacionamos tal achado ao seguinte contexto: informação da importância do uso da MECG e acompanhamento no ambulatório especializado. Os principais motivos para o não uso foram a questão financeira, o relato de dor e a falta de conhecimento da indicação do uso.

\section{REFERÊNCIAS}

1. Medeiros J, Mansilha A. Estratégia terapêutica na doença venosa crônica. Angiol Cir Vasc. 2012;8(3):110-26.

2. França LHG, Tavares V. Insuficiência venosa crônica: uma atualização. J Vasc Bras. 2003;2(4):318-28.

3. Ayala-García MA, Reyes JS, Muñoz Montes N, Guaní-Guerra E. Frequency of use of elastic compression stockings in patients with chronic venous disease of the lower extremities. Phlebology. 2019;34(7):481-5. http://dx.doi.org/10.1177/0268355518822356. PMid:30621524.

4. Benigni JP, Sadoun S, Allaert FA, Vin F. Efficacy of Class 1 elastic compression stockings in the early stages of chronic venous disease: a comparative study. Int Angiol. 2003;22(4):383-92. PMid:15153823.

5. Figueiredo MAM, Castro AA, Simões R. Projeto diretrizes: terapia de compressão de membros inferiores. São Paulo: Sociedade Brasileira de Angiologia e Cirurgia Vascular; 2011. 10 p.

6. Marcondes AM, Figueiredo AD, Cabral AL. Avaliação do efeito da meia elástica na hemodinâmica venosa dos membros inferiores de pacientes com insuficiência venosa crônica. J Vasc Bras. 2004;3(3):231

7. Escudero Rodríguez J-R, Fernández Quesada F, Bellmunt Montoya S. Prevalence and clinical characteristics of chronic venous disease in patients seen in primary care in Spain: results of the International Study Vein Consult Program. Cir Esp. 2014;92(8):53946. PMid:24355629.

8. Castro e Silva M, Cabral A, Barros JN, Castro AA, Santos MERC, Teixeira AR. Diagnóstico e tratamento da Doença Venosa Crônica. J Vasc Bras. 2005;4(3):185-204.

9. Raju S, Neglén P. Chronic venous insufficiency and varicose veins. N Engl J Med. 2009;360(22):2319-27. http://dx.doi.org/10.1056/ NEJMcp0802444. PMid:19474429. 
10. Figueiredo $M$. A terapia da compressão e sua evidência científica. J Vasc Bras. 2009;8(2):100-2. http://dx.doi.org/10.1590/ S1677-54492009000200002.

11. Vayssairat $M$, Ziani E, Houot B. Efficacité versus placebo de la contention classe 1 dans l'insuffisance veineuse chronique des membres inférieurs. J Mal Vasc. 2000;25(4):256-62. PMid:11060420.

12. Raju S, Hollis K, Neglen P. Use of compression stockings in chronic venous disease: patient compliance and efficacy. Ann Vasc Surg 2007;21(6):790-5. http://dx.doi.org/10.1016/j.avsg.2007.07.014. PMid:17980798.

13. Ayala-García MA, Reyes JS, Muñoz Montes N, Guaní-Guerra E. Frequency of use of elastic compression stockings in patients with chronic venous disease of the lower extremities. Phlebology. 2019;34(7):481-5. http://dx.doi.org/10.1177/0268355518822356 PMid:30621524.

14. Ziaja D, Kocełak P, Chudek J, Ziaja K. Compliance with compression stockings in patients with chronic venous disorders. Phlebology. 2011;26(8):353-60. http://dx.doi.org/10.1258/phleb.2010.010086. PMid:21810940.

15. Moffatt CJ. Factors that affect concordance with compression therapy.J Wound Care. 2004;13(7):291-4. http://dx.doi.org/10.12968/ jowc.2004.13.7.26641. PMid:15977771.

16. Moffatt CJ. Perspectives on concordance in leg ulcer management. J Wound Care. 2004;13(6):243-8. http://dx.doi.org/10.12968/ jowc.2004.13.6.26894. PMid:15214144.
Correspondência Francisco Eduardo Coral Rua Campos Sales, 1085, apartamento 601C CEP 80030-378 - Curitiba (PR), Brasil

Tel.: (41) 99972-0172

E-mail:coral.francisco@gmail.com

Informações sobre os autores FEC - Mestre em Cirurgia, Universidade Católica do Paraná (PUCPR); Especialista em Cirurgia Vascular, Sociedade Brasileira de Angiologia e de Cirurgia Vascular (SBACV); Chefe, Serviço de Cirurgia Vascular e

Endovascular, Hospital da Irmandade da Santa Casa de Curitiba. GGG - Especialista em Cirurgia Vascular, Sociedade Brasileira de Angiologia e de Cirurgia Vascular (SBACV); Cirurgiã Vascular, Hospital da Irmandade da Santa Casa de Curitiba.

APC, ALMR, MMG e HP - Graduados em Medicina, Universidade Católica do Paraná (PUCPR).

Contribuições dos autores Concepção e desenho do estudo: FEC, GGG Análise e interpretação dos dados: FEC, GGG, APC, ALMR Coleta dos dados: ALMR, HP, APC, MMG Redação do artigo: APC, ALMR Revisão crítica do texto: FEC, GGG Aprovação final do artigo*: FEC Análise estatística: FEC, GGG, estatista externo Responsabilidade geral pelo estudo: FEC

*Todos os autores leram e aprovaram a versão final submetida ao J Vasc Bras. 